African Journal of Biomedical Research, Vol. 8 (2005); 157 - 162 ISSN 1119 - 5096 @ Ibadan Biomedical Communications Group Available online at http://www.bioline.org.br/md

Full Length Research Article

\title{
Mycelial Growth and Antibacterial Metabolite Production by Wild Mushrooms
}

\author{
${ }^{1 *}$ Shittu, O.B; ${ }^{2}$ Alofe, F.V ; ${ }^{3}$ Onawunmi, G.O; ${ }^{4}$ Ogundaini, A.O; and Tiwalade, T.A. ${ }^{4}$ \\ ${ }^{*}$ Department of Microbiology, University of Agriculture, Abeokuta and Departments of \\ ${ }^{2}$ Microbiology, ${ }^{3}$ Pharmaceutical Microbiology and Pharmaceutical Chemistry, Obafemi \\ Awolowo University, Ile-Ife,
}

Received: May, 2005

Accepted: September, 2005

\begin{abstract}
Russula sp. and Pycnoporus cinnabarinus (wild mushrooms) were subjected to laboratory cultivation by spore germination and tissue culturing on Sabouraud dextrose agar plates. Subsequently, the growth and production of metabolite(s) were monitored in submerged fermentation for 7days using agar diffusion method. The result obtained showed that metabolite production peaked on the fourth day in Russula sp. and on the fifth day in Pycnoporus cinnabarinus with subsequent decrease in activity of the fermentation extract. Dry weight increases with fermentation time in both mushrooms.
\end{abstract}

Keywords: Wild mushroom, Spore germination, Tissue culturing, Antibacterial metabolite

\section{INTRODUCTION}

Some mushrooms contain compounds, which can make a contribution to the general health of man (Elliot, 1997). As mushrooms are widely distributed all over the world, some of them have been used in traditional medicine as antiinflammatory, analgesics, hemostatic, diuretic, nourishment, antibechic and antitumour agents (Koyama et al., 1997).

Most of the medicinal extracts from mushrooms are different forms of polysaccharides and all of them are strengtheners of the immune system with little or no side effects (Oei, 1991, 1996; Gao et al., 1997). For example, a sizofiran, antitumour polysaccharide extracted from the culture broth of Schizophyllum commune is an effective immuno-therapeutic agent for cervical carcinoma because it stimulates a rapid recovery of the immunological status impaired by radiotherapy (Miyazaki et al., 1995). Lentinus edodes is another immunological strengthener.
Some mushrooms are used for the treatment of gastric ulcer, duodenal ulcer and chronic gastritis. A good example is Hericium erinacius (Oei, 1991, 1996). Some mushrooms such as Tremella fulciformis are used for curing leukaemia, coughing, phlegm and asthma of patients suffering from chronic bronchitis (Oei, 1991, 1996). According to Gao et al. (1997) mushrooms like $T$. fulciformis also possesses antitumour, hypoglycaemic and cytosine stimulating activities. Antinoceptive components have been isolated from Ganoderma lucidum (Koyama et al., 1997). This fungus ( $G$. lucidum) has been used in curing neurasthenia, chronic bronchitis and coronary heart disease. Lanostanes triterpenes is another compound from mushroom extracts that have been found to be of medicinal value. The compound has been isolated from G. lucidum and it has cytotoxic, antineoplastic and hypotensive activities. The fungus is widely used in Asian medicine to treat all types of diseases (Keller et al., 1997). Another collagenase inhibitor 
considered as candidate for medicine for the treatment of rheumatism, metastasis or periodontal disease has been isolated from Daedalea dickinsii (Kawagishi et al., 1997). Oyster mushrooms (Pleurotus ostreatus) decrease serum and liver cholesterol and increases cholesterol 7-hydroxylase activities and faecal excretion of neutral sterols and bile acids in hypercholesterolomic rats (Bobek et al., 1994). Engler et al. (1998) obtained antibiotic metabolites named oudemasin $A$ and $D$, illudin $S$ and pterulone B from Collybia nivalis, Omphalotus olearius, a Flavolaschia and a Pterula sp.

A strain of Cyathus striatus has been found to produce striatins $A, B, C$ during mycelial growth and these crystalline antibiotics were highly active against Fungi imperfecti and a variety of Grampositive bacteria, as well as some Gram negative bacteria (Anke et al., 1976). Lactarii vellutinus produces an extremely labile compound, identified as stearyl-vellutinal when injured. This biologically inactive precursor which has been shown to be responsible for the dark blue color that develops when the mushroom is treated with sulphovanillin (Favre-Bonvin et al., 1982) is rapidly converted to a strongly antibiotic and pungent sesquiterpenoids dialdehydes such as isovelleral [2]. (Sterner et al., 1983; Stadler and Sterner, 1998).

Various bioactive compounds isolated from culture extracts of Ethiopian higher fungi showed other biological properties such as antiprotozoal, anthelmintic, phytotoxic and brine shrimp lethality activities (Dagne and Abate, 1995). Also, Inchausti et al. (1997) investigated Leishmanicidal and Trypanocidal activity of the extracts and secondary metabolites of some Basidiomycetes. A naturally occurring purine nucleoside found in some mushrooms showed high degree of activity against Mycobacterium (Gupta and Bhakum, 1982). Aqueous extracts of spent mushroom substrate are used in foliar disease control (Yokalem et al., 1994).

Wild mushrooms are seasonal, and a particular mushroom may disappear from the initial place of collection for a number of years, appearing in another place beyond reach (Harding, 1996; Laessoe, 1998).

Russula sp. (gilled mushroom) and Pycnoporus cinnabarinus (polypore) are wild mushrooms. Their fruitbodies were screened and found to possess antibacterial properties in previous work (Fajana et al., 1999). This study examined the two wild mushrooms in laboratory cultivation for growth and bioactive compound production, in submerged fermentation.

\section{MATERIALS AND METHODS}

\section{Cultivation experiments}

Mycelial cultivation through spore germination Sabouraud dextrose agar slant was heavily inoculated with spores collected from the gilled mushroom, Russula sp. Incubation was carried out at ambient temperature for 7 days. Several subculturing exercises were carried out until a pure culture was obtained. The mycelial culture thus obtained was used as inoculum in subsequent experiments.

\section{Mycelial cultivation through tissue culturing}

The fruitbody of the polypore mushroom Pycnoporus cinnabarinus was collected at a young stage with the aid of sterile forceps, wrapped with sterile foil paper and transported to the laboratory. It was washed thoroughly with several changes of sterile distilled water and was, thereafter, aseptically broken lengthwise exposing the inner tissue (trama) with the aid of a sterile blade. A small piece of $2 \times 2 \mathrm{~mm}$ of the sterile tissue was then aseptically transferred onto plates of solid acidified Sabouraud dextrose agar. Four replicates were made and the plates were incubated at ambient temperature for 72 hours. Subculturing for pure tissue mycelial production was prepared by transferring a small square of $5 \mathrm{x}$ $5 \mathrm{~mm}$ from the mother plate onto a fresh solid media plates. All transfers were made aseptically. The tissue culture obtained thus was used in subsequent experiments.

\section{Measurement of linear growth}

Colonies were grown at ambient temperature. The colony diameters were measured every 24 hours to the nearest $0.5 \mathrm{~mm}$. Mean diameter of six colonies at 24 hourly for 7 days were fitted to the best straight line by regression analysis and the slope of the best fit line was taken as the rate.

\section{Cultivation for metabolite production Growth conditions for metabolite production}

Fungal cultures were grown in $100 \mathrm{ml}$ conical flasks each of which contained $50 \mathrm{ml}$ of sterile Sabouraud dextrose broth. The cultures were then incubated at room temperature for 7 days by shaking on a rotatory shaker (Gallenkhamp) at $120 \mathrm{rev} / \mathrm{min}$.

\section{Measurements of growth for metabolite production}

Cultures of each of the organisms were withdrawn 24 hourly in triplicates and filtered using No.1 filter 
paper. The mycelia on the pre-weighted filter paper were oven-dried at $80^{\circ} \mathrm{C}$ to a constant weight. The weights were used to plot the growth characteristics.

\section{Isolation of the crude extracts}

The filtrates obtained 24 hourly in triplicates were pooled together and partitioned in ethyl acetate using a separating funnel. The ethyl acetate fractions were evaporated to dryness in vacuo. For zero hour extracts, a negative control flask (not inoculated with fungal culture) and a positive control flask (inoculated with fungal culture) were extracted within an hour of inoculation.

\section{Bioassay monitoring of antibacterial metabolite production by agar diffusion method.}

The extracts were reconstituted in $0.5 \mathrm{ml}$ of $50 \%$ aqueous methanol and $100 \mu \mathrm{l}$ of each test solution was assayed using agar cup plate diffusion method earlier described in Fajana et al. (1999). The culture plates were then sprayed with methyl thiazoyl tetrazolium chloride (MTT), and further incubated for 15 minutes. The diameters of zones of inhibition were then measured with a transparent ruler.

\section{RESULTS}

Measurement of linear growth on solid media The results obtained for the linear growth of Russula sp. and Pycnoporus cinnabarinus are shown in fig.1. The rate of growth as shown from the slope is $1.23 \mathrm{~mm}$ per hour for Russula sp and $0.54 \mathrm{~mm}$ per hour for Pycnoporus cinnabarinus. The growth on Sabouraud dextrose agar is shown in plate 1.

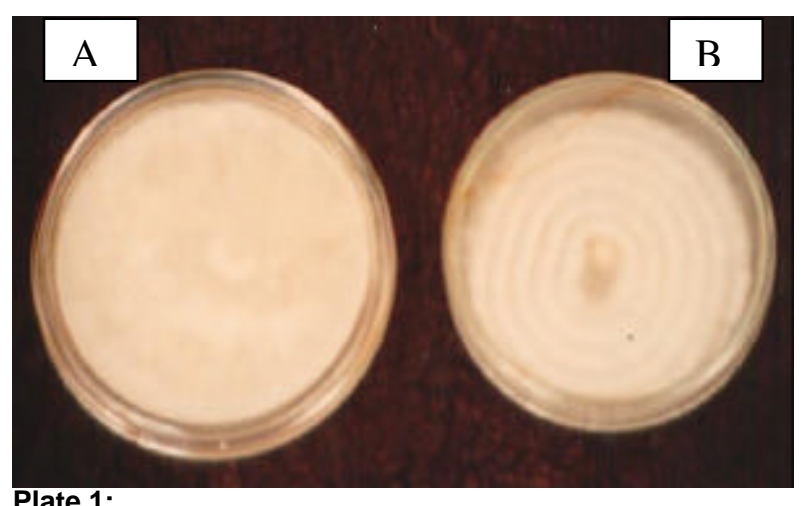

Cultures of Russula sp. and Pycnoporus cinnabarinus grown at ambient temperature for 7days on Sabouraud Dextrose Agar. $\mathrm{A}=$ Russula sp. $; \quad \mathrm{B}=$ Pycnoporus cinnabarinus

- Pycnoporus cinnabarinus

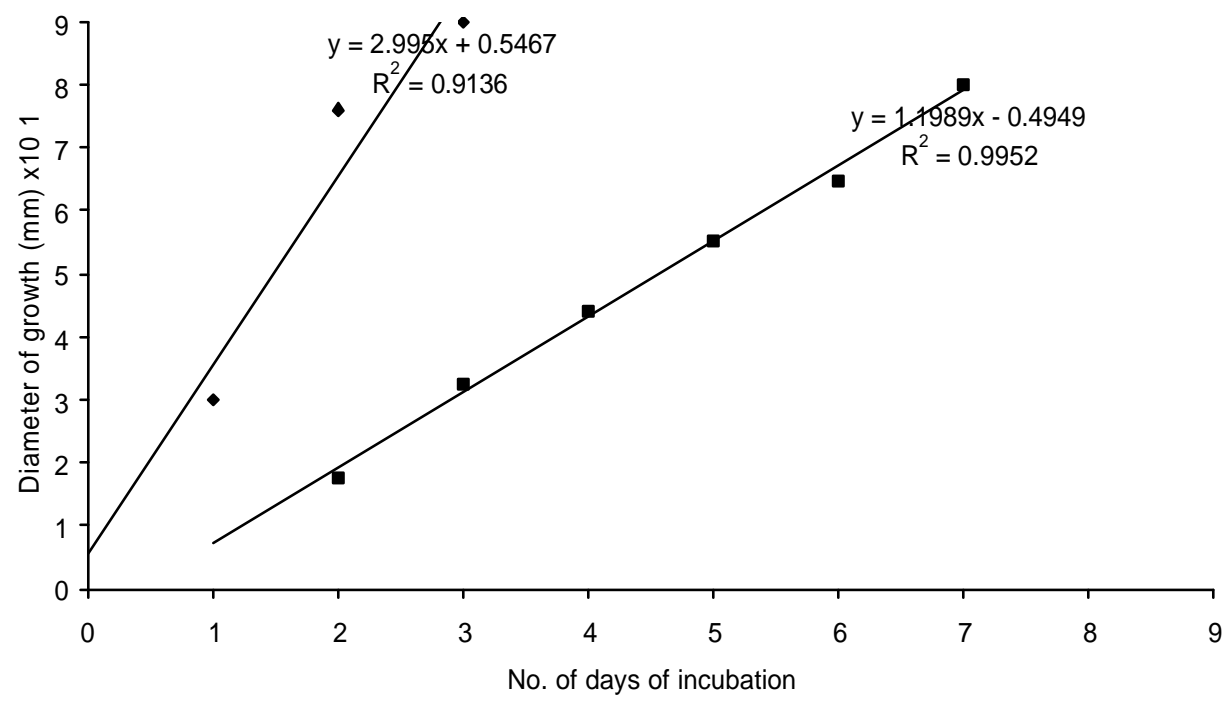

Fig. 1. Linear growth of mushroom on sabouraud dextrose agar 


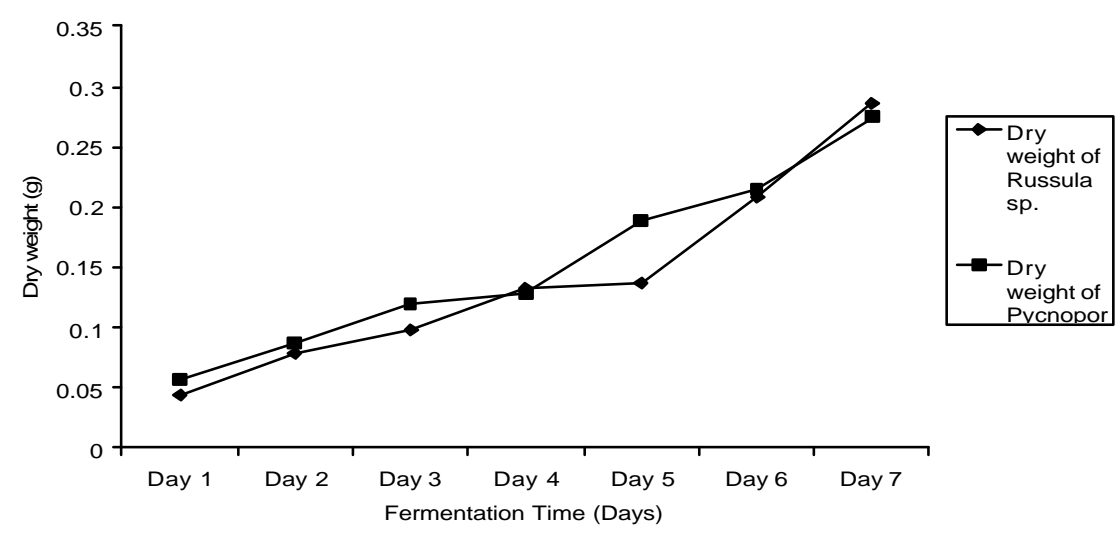

Fig. 2. Growth characteristics of Mushrooms

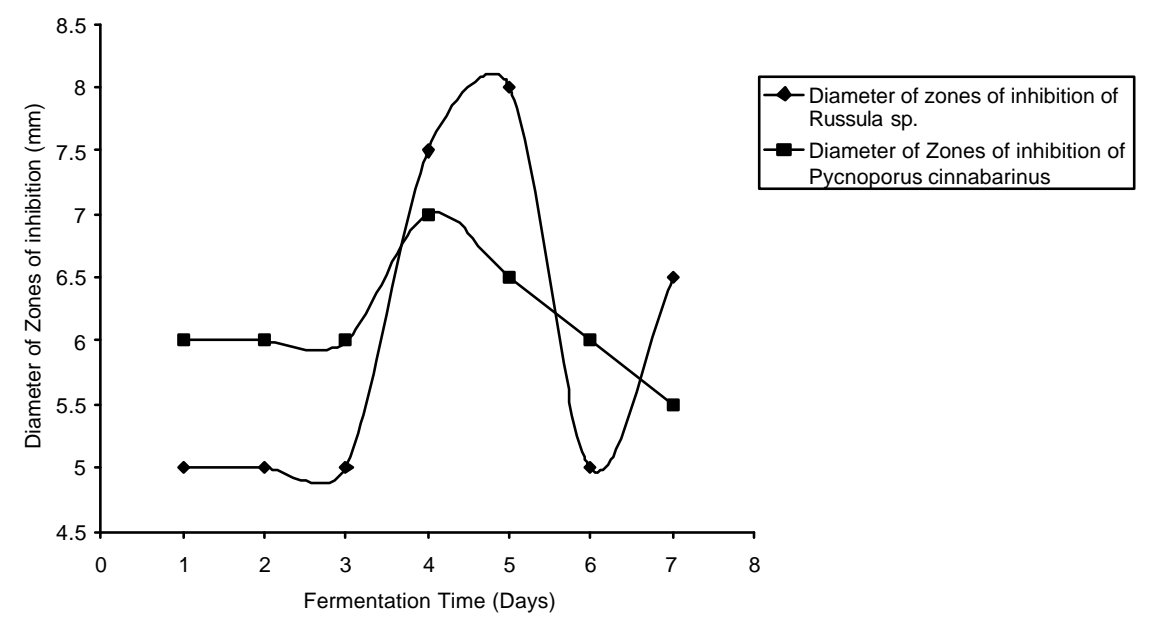

Fig. 3. Production of Bioactive compound by mushroom

Growth characteristics on Sabouraud dextrose liquid medium.

The inocula of both mushrooms formed filamentous strands and numerous pellets that were of different sizes. The pellets were covered with tiny, short, and pointed hairs.

\section{Antibacterial test of aqueous fraction of culture filtrate}

The antibacterial test of the aqueous fraction obtained after extraction of the culture filterate with ethyl acetate revealed no activity.

Measurement of growth and antibacterial metabolite production

Growth characteristics by dry weight

The growth characteristics by dry weight of Russula sp. and Pycnoporus cinnabarinus as shown in Fig. 2 showed relative increase in mycelial mass in relation to fermentation time.

Bioassay monitoring of antibacterial metabolite production

The results of the monitoring of the production of bioactive compound in the culture filtrate of the extracts of submerged fermentation by agar diffusion method for Russula sp. and Pycnoporus cinnabarinus are shown in Figs. 3 respectively. The peak of metabolite production as detected by the activity in Russula sp was on the fifth day. The actual production, as deduced from the graph, was between the fourth and the fifth day, subsequently followed by a decrease and then a rise again in activity. For Pycnoporus cinnabarinus, the peak of the activity was on the fourth day, with the actual production between the third and fourth day. This was followed by gradual decrease in activity. 


\section{DISCUSSION}

The mycelia of Russula sp. and Pycnoporus cinnabarinus were successfully grown on conventional laboratory media. Russula $s p$. was cultivated by spore germination method while Pycnoporus cinnabarinus was cultivated through tissue culturing method. In practice, tissue culturing is regarded as the best method of mushroom cultivation (Oei, 1996). It has been reported that spore germination has the disadvantage of taking longer period, with the minute spore size making it relatively difficult to handle (Yu et al., 1984; Oei, 1996). However, from this study, it was observed that the spores of Russula $s p$. germinated within the seven days of incubation. The mycelia of Russula $s p$. had a rate of growth of $1.23 \mathrm{~mm} /$ hour while that of Pycnoporus cinnabarinus had a rate of growth of $0.53 \mathrm{~mm} /$ hour (Fig.1) indicating a higher rate of growth for the Russula sp. Spore germination and growth measurement are biological assay indicating the viability of the fungal spores and hyphae (Yu et al., 1984).

The growth and production of antibacterial metabolite of Russula sp. and Pycnoporus cinnabarinus as shown in Figs. 2 and 3 revealed that dry weight increases with fermentation time while activity was detected in all the fermentation extract. The activity resulting from the first three days could be attributed to an inhibitory effect of the fermentation broth as indicated from the zero day (control) activities. This also revealed that the culture media extract itself exhibited an antimicrobial activity against $B$. subtilis NCTC 8236 , which is another form of interference in the activity being measured. The interference could be suggested to have arisen from certain ethyl acetate extractable organic component(s) of the broth, which had become concentrated by extraction, to possibly a growth inhibitory concentration.

For both Russula sp. and Pycnoporus cinnabarinus, the results observed on the production of antibacterial metabolite showed that, by the fourth day, activity was at the peak or very near the peak (Figs. 3). The fall in activity in both species suggests that the active component is chemically unstable or is being used up by degradation or further converted to other metabolites. The subsequent rise in activity of Russula sp. suggests that other metabolite, which is also active, is being formed.

This study had shown the pattern of growth and antibacterial metabolite production by mushrooms collected from the wild. Such a study should be

a preliminary experiment, which will help in the location of the appropriate harvest time in submerged fermentation studies.

\section{REFERENCES}

Anke, T., Oberwinkler, F., Steglich, W. and Hofle, G. (1976): The striatins-New antibiotics from Basidiomycete Cyathus striatus (HUDS.ex.PERS) WILLD. The Journal of Antibiotics XXX (3): 221-225.

Bobek, P., Ondreika P., KIvanova, J. and Ozdin, L. (1994): Oyster mushrooms (Pleurotus ostreatus) decreases serum and liver cholesterol and increase cholesterol $7 \alpha$-hydroxylase activity and faecal excretion of neutral sterols and bile acids in hypercholesterolomic rats. Nutritional Research 14(11): 1683-1688.

Dagne, E. and Abate, D. (1995): Bioactive compounds from plants and higher fungi of Ethiopia. In; Phytochemistry of plants used in traditional medicine. Hostettmann, K., Marston, A., Millard, M. and Harmburger, M.eds. Oxford University Press, pp. 295-312.

Elliot, T. (1997): Mushrooms. Society for General Microbiology Quarterly24(1): 8-9.

Engler, M., Anke, T. Sterner, O. (1998): Production of antibiotics by Collibia nivalis. Omphalotus olearuius, a Favolaschia and a Pterula species on natural substrates. Zeitschrift fur Naturforschung, Section C. Biosciences 53 (516): 318-324.

Fajana, O.B. Alofe, F.V. Onawunmi, G.O. Ogundaini, A.O. and Tiwalade, T.A. (1999): Antimicrobial studies on Nigerian higher fungi. Nigerian Journal of Natural Products and Medicine 3: 64-65.

Favre-Bonvin, J. K., Gluchof-Fiasson, K. and Bernillion, J. (1982): Structure du stearylvellutinal, sesquiterpenoide natural de Lactarius vellutinus Bert. Tetrahedron Letters 23: 19071908.

Gao, Q; Killie, M. K.; Chen, H.; Jiang, R. and Seljelid, R. (1997): Characterisation and cytokinestimulating activities of acidic heteroglycans from Tremella fuciformis. Planta medica 63:457-460.

Gupta, P. K. and Bhakum, D. S. (1982): Synthesis of 9- $\beta$-D-ribofuranosyl purine (Nebularin) and its analogs. Indian Journal of Chemistry 20(7): 534-537.

Harding, P. (1996). Mushrooms and toadstools photoguide. Harper Collins publishers, Glasgow. P. 256.

Inchausti, A. Yaluff, G., rojas de Arias, A. Torres, S., Ferreira, M. E., Nakayama, H., 
Schinini, A., Lorenzen, K., Anke, T. and Fournet, A. (1997): Leishmanicidal and trypanocidal activity of extracts and secondary metabolites from basidioycetes. Phytotherapy Research 11: 193-197.

Kawagishi, H. L., Li, H. Tanno, O., Inoue, S. Ikeda, S., Ohnishi-Kameyama, M. and Nagata, T. (1997): A lanostane-type Tripterpene from a mushroom Daedelea dickinsii. Phytochemistry 46(5): 959-961.

Keller, A. C., Keller, J., Maillard, M. P. and Hostettmann, K. (1997): A lanostane-type steroid from the fungus, Ganoderma carnosum. Phytochemistry 40(5): 963-969.

Koyama, K., Imaizumi, T., Akiba, M., Kinoshita, K., Suzuki, A., Yano, S., Watanabe, K. and Naoi, Y. (1997): Antinoceptive components of Ganoderma lucidum. Planta Medica 63: 224-227.

Laessoe, T.1998. Eye withness handbooksMushroom. Dorling Kindersley Ltd. London. Great Britin, pp.304.

Miyazaki, K., Mizutani, H., Katauchi, H., Fukumaka, K., Fujisaki, S. and Okamura, H. (1995): Activated (HLA-DR+) T-lymphocyte subsets in cervical carcinoma and effects of Radiotherpay and immunotherapy with sizofiram on cell-mediated immunity and survival. Gynaecologic Oncology 56: 412-420.

Oei, P. (1991): Manual on mushroom cultivation. Techniques, species and opportunities for commercial applications in developing countries. TOOL publications, Amsterdam, Netherlands. P243.

Oei, P. (1996): Mushroom cultivation. With special emphasis on appropriate techniques for developing countries. TOOL publications, Leiden, Netherlands. P256.

Stadler, M. and Sterner, O. (1998): Production of bioactive secondary metabolites in the fruitbodies of a macrofungi as a response to injury. Phytochemistry 49: 1013-1019.

Sterner, O. Bergman, R., Kesler, E., Nilson, L. Oluwadiya, J. and Wickberg, B. (1983): Velutinal esters of lactarius vellereus and $L$. nector: The preparation of free velutinal. Tetrahedron letters 24: 1415-1418.

Yokalem, D. S., Harris, R. F. and Andrews, J. H. (1994): Aqueous extracts of spent mushroom substrate for foliar disease control. Compost Science and Utilization 2(4): 67-74.

Yu, S. Q., Trione, E.J. and Ching, T. M. (1984): Biochemical determination of the viability of fungal spores and hyphae. Mycologia 76(4): 608-613.

*Author to whom correspondence should be addressed. Olufunke_b@yahoo.com 